\title{
Clinical efficacy of gangliosides on premature infants suffering from white matter damage and its effect on the levels of IL-6, NSE and S100ß
}

\author{
XIAOPENG WANG ${ }^{*}$, XIUYING TIAN* ${ }^{*}$, JUNLING MA and JUN ZHENG \\ Department of Neonatology, Tianjin Central Hospital of Obstetrics and Gynecology, \\ Tianjin 300100, P.R. China
}

Received May 16, 2018; Accepted January 30, 2019

DOI: $10.3892 /$ etm.2019.7539

\begin{abstract}
This study investigated the clinical efficacy of gangliosides on premature infants suffering from white matter damage and its effect on the levels of IL-6, neuron-specific enolase (NSE) and S100 $\beta$. Seventy-six cases of premature infants suffering from white matter damage admitted to the Tianjin Central Hospital of Obstetrics and Gynecology from February 2016 to March 2017 were enrolled in this study. They were randomly divided into the control group and the observation group with 38 cases in each group. Control group was given conventional treatment, while the observation group was given ganglioside treatment on the basis of the treatment given to the control group. Craniocerebrum ultrasonic detection was used to observe the condition of white matter around the ventricle of child patients in the two groups, before and after treatment. ELISA was used to detect the levels of IL-6, NSE and S100 $\beta$. Gesell developmental scale was used to compare the developmental quotient (DQ) of various function regions of the children. The total effective rate of the observation group was higher than that of the control group $(\mathrm{P}<0.05)$. The gray value of craniocerebrum ultrasonic detection in the observation group was significantly lower than that in the control group $(\mathrm{P}<0.05)$. IL-6, S100 $\beta$ and NSE levels of the child patients in the two groups were significantly declined at 7 and 14 days after birth $(\mathrm{P}<0.05)$. After 1 year, the observation group scored significantly higher DQ than the control group in the aspects of social adaptation, gross motor, fine motor, language and personal social contact. The
\end{abstract}

Correspondence to: Dr Jun Zheng, Department of Neonatology, Tianjin Central Hospital of Obstetrics and Gynecology, 156 Three Road, Nankai, Tianjin 300100, P.R. China

E-mail:nizi86945@163.com

${ }^{*}$ Contributed equally

Key words: gangliosides, white matter damage, premature infants, IL-6, neuron-specific enolase, S100 $\beta$ sequel incidence of patients in the observation group was significantly lower than that of the control group $(\mathrm{P}<0.05)$. In conclusion, the intervention treatment with ganglioside for premature infants suffering from white matter damage was beneficial and provided a protective effect. It also reduced sequel and produced some promising results.

\section{Introduction}

Brain white matter damage is a relatively common neurological disease. Along with the increasing improvement of medical technologies, the technologies of obstetrics and neonate intensive care have also been improved. Therefore, the survival rate of premature infants has been obviously increased. However, the incidence of brain damage of premature infants is also gradually increasing (1). Brain white matter damage is one of the main neuropathological forms of brain damage of premature infants. It can influence their intelligence development and cause sequela of nervous system, such as dysnoesia and cerebral palsy. As a result, heavy burden falls on the family of the children and society in general (2). Premature infants suffering from white matter damage do not show specific clinical symptoms or signs at early stage, and it is hard to diagnose the problem simply by clinical manifestations. Besides, no effective intervention measures have been developed yet. Therefore, it is crucial to find an effective treatment or prevention method in order to improve patients' living quality (3). IL-6 has been shown to have neurotrophic and neuroprotective effects as well as neurotoxic effects on the central nervous system (4). S100 $\beta$ protein and serum neuron-specific enolase (NSE) are among the well-known markers of brain damage. They can reflect the degree of the brain damage as well as the neurological function damage (5). Ganglioside are a kind of essential glycolipids of ganglion series for the nerve growth and brain development and promote nerve regeneration (6). In the present study, by giving ganglioside to premature infants suffering from white matter damage, we aimed to show its possible efficacy and to analyze the effect of ganglioside on IL-6, NSE and S100 $\beta$ levels with the expectation to provide a basis for the treatment on premature infants suffering from white matter damage. 
Table I. Comparison of baseline data between the child patients in the two groups.

\begin{tabular}{lcccc}
\hline Items & Control group (n=38) & Observation group $(\mathrm{n}=38)$ & $\mathrm{t} / \chi^{2}$ value & P-value \\
\hline Child patient sex (male/female) & $20 / 18$ & $22 / 16$ & 0.053 & 0.818 \\
Fetal age (weeks) & $29-35$ & $28-36$ & & \\
Average fetal age (weeks) & $33.18 \pm 1.25$ & $33.36 \pm 1.36$ & 0.601 & 0.550 \\
Child patient weight (kg) & $2.13 \pm 0.28$ & $2.12 \pm 0.32$ & 0.145 & 0.885 \\
Brain white matter damage [n (\%)] & & & & \\
$\quad$ Light damage & $27(71.05)$ & $29(76.32)$ & 0.068 & 0.795 \\
Medium or serious damage & $11(28.95)$ & $9(23.68)$ & & \\
\hline
\end{tabular}

\section{Patients and methods}

General data. From February 2016 to March 2017, 76 cases of premature infants suffering from white matter damage were selected using a random sampling method. The inclusion criteria were i) meeting the diagnostic criteria of white matter damage (7); ii) premature infants; and iii) informed consent forms signed by parents. Exclusion criteria were i) term infants; and ii) patients allergic to the medicine used in this research. The study was approved by the Ethics Committee of Tianjin Central Hospital of Obstetrics and Gynecology (Tianjin, China) and informed consents were signed by the guardians of the child patients. There were respectively 38 cases in the observation and control group. Comparison of the general data between the children in the two groups showed that they were not significantly different $(\mathrm{P}>0.05)$ (Table I).

Treatment. Control group was given conventional treatment, which included warmth retention, reasonable oxygen supply and scientific feeding. Good ventilation was maintained for patients and their blood gas and $\mathrm{pH}$ values were kept within the normal range. Peripheral region and various organs were kept with enough hemoperfusion, and the heart rate and blood pressure were kept within the normal range. Blood glucose was maintained at normal high value $(5.0 \mathrm{mmol} / \mathrm{l})$ to ensure the metabolic needs of neurons. Medical staff closely supervised any change of the disease condition of the children. Occurrence of any abnormality was taken care of by symptomatic treatment. The observation group was given ganglioside (SFDA approval no. H20066833; Changchun Xiangtong Pharmacy, Co., Ltd., Xiangtong, China). The treatment plan was as follows: $20 \mathrm{mg}$ of intravenous drip every day, 14 days as one course and 1-3 courses should be given based on the disease condition of the children.

Test of relative indexes. GE Voluson 730 Expert ultrasonic testing instrument [GE Healthcare (China) Co., Ltd., Beijing, China] was used for craniocerebrum ultrasonic detection at 1, 7 and 14 days after birth (transducer frequency, $5-9 \mathrm{mHz}$ ). Coronal and sagittal scans were conducted via bregma. Images at the central part and occipital horn of lateral ventricle were observed. Abnormal echo of white matter was taken as abnormity.

Central venous blood $(2 \mathrm{ml})$ was extracted from children before and after treatment. The supernatant was collected after centrifugation at $3,000 \mathrm{x}$ g for $10 \mathrm{~min}$ at $4^{\circ} \mathrm{C}$ and kept at $-70^{\circ} \mathrm{C}$. ELISA was used to measure IL-6, NSE and S100 $\beta$ levels. The relative kits were provided by the R\&D Systems, Inc. (Richmond, CA, USA) and were used according to the manufacturer's instructions. An enzyme-labeling instrument (Jiangsu Potebio Co., Ltd., Jiangsu, China) was used to read OD values (wavelength, $450 \mathrm{~nm}$ ) and to calculate the concentrations of IL-6, NSE and S100ß.

Evaluation methods. The following degrees were used to evaluate the white matter damage (7): i) light: by ultrasonic detection, the echo intensity of white matter around ventricle of the patients was weaker than that of choroid; ii) medium: ultrasonic results showed that the echo intensity of white matter around ventricle was the same as that of the choroid plexus; iii) serious: ultrasonic results showed that the echo intensity of white matter around ventricle was stronger or the same as that of the choroid plexus, but the range exceeded the triangle area of lateral ventricle.

Craniocerebrum ultrasonic detection was used to observe the condition of white matter around the ventricle of the children in the two groups, before and after treatment. ELISA was used to detect the concentration levels of IL-6, NSE and S100 $\beta$. Gesell developmental scale (8) was used to evaluate the developmental quotient (DQ) of various function regions of the children in the two groups at 12 months after treatment (9). Function regions include social adaptation, gross motor, fine motor, language, and personal social contact.

Statistical analysis. SPSS 19.0 (IBM Corp., Armonk, NY, USA) was used for data analysis. Measurement data were expressed as mean \pm standard deviation (mean \pm SD) and were compared by t-test; enumeration data were expressed as percentage and compared by $\chi^{2}$ test. Treatment effect was evaluated by a rank sum test. ANOVA was used for multiple group comparisons with Dunnett's post hoc test. $\mathrm{P}<0.05$ was considered to indicate a statistically significant difference.

\section{Results}

Comparison of the efficacy on child patients in the two groups showed that the total effective rate in the observation group was significantly higher than that in the control group $(\mathrm{P}<0.05)$ (Table II).

Comparison of the gray values of craniocerebrum ultrasonic detection of the children in the two groups showed that 
Table II. Comparison of efficacy on child patients in the two groups [n (\%)].

\begin{tabular}{lcccrc}
\hline Group & $\mathrm{n}$ & Obviously effective & Effective & Not effective & Total effective \\
\hline Observation group & 38 & $26(68.42)$ & $7(18.42)$ & $5(13.16)$ & $33(86.84)$ \\
Control group & 38 & $12(31.58)$ & $11(28.95)$ & $15(39.47)$ & $23(60.53)$ \\
\hline
\end{tabular}

The results from the Rank sum test of the efficacy on child patients of the two groups were $\mathrm{Z}=3.176, \mathrm{P}=0.018$.

Table III. Comparison of gray values of craniocerebrum ultrasonic detection of child patients in the two groups (mean \pm SD).

\begin{tabular}{lcccr}
\hline Group & $\mathrm{n}$ & 1 day after birth & 7 days after birth & 14 days after birth \\
\hline Observation group & 38 & $126.67 \pm 3.23$ & $105.35 \pm 3.28^{\mathrm{a}}$ & $96.68 \pm 3.24^{\mathrm{a}}$ \\
Control group & 38 & $126.27 \pm 3.64$ & $125.47 \pm 3.36$ & $125.83 \pm 3.78$ \\
t value & & 0.507 & 26.414 & 36.093 \\
P-value & & 0.614 & $<0.001$ & $<0.001$ \\
\hline
\end{tabular}

${ }^{\text {aP }}<0.05$, compared to the 1 st day after birth. $\mathrm{SD}$, standard deviation.

Table IV. Comparison of IL-6 levels of child patients in the two groups ( $\mathrm{pg} / \mathrm{ml}$, mean $\pm \mathrm{SD})$.

\begin{tabular}{lcccc}
\hline Group & $\mathrm{n}$ & 1 day after birth & 7 days after birth & 14 days after birth \\
\hline Observation group & 38 & $26.63 \pm 3.23$ & $20.35 \pm 3.28^{\mathrm{a}}$ & $18.38 \pm 3.24^{\mathrm{a}}$ \\
Control group & 38 & $26.58 \pm 3.64$ & $23.47 \pm 3.36^{\mathrm{a}}$ & $21.83 \pm 3.78^{\mathrm{a}}$ \\
t value & & 0.063 & 4.096 & 4.272 \\
P-value & & 0.950 & $<0.001$ & $<0.001$ \\
\hline
\end{tabular}

${ }^{\text {aP }}<0.05$, compared to the 1 st day after birth. $\mathrm{SD}$, standard deviation.

Table V. Comparison of NSE levels of child patients in the two groups (ng/ml, mean \pm SD).

\begin{tabular}{lcccc}
\hline Group & $\mathrm{n}$ & 1 day after birth & 7 days after birth & 14 days after birth \\
\hline Observation group & 38 & $6.62 \pm 1.18$ & $4.64 \pm 0.78^{\mathrm{a}}$ & $3.52 \pm 0.47^{\mathrm{a}}$ \\
Control group & 38 & $6.65 \pm 1.13$ & $5.32 \pm 0.61^{\mathrm{a}}$ & $5.03 \pm 0.34^{\mathrm{a}}$ \\
t value & & 0.113 & 4.233 & 16.046 \\
P-value & & 0.910 & $<0.001$ & $<0.001$
\end{tabular}

${ }^{\mathrm{a}} \mathrm{P}<0.05$, compared to the 1 st day after birth. NSE, neuron-specific enolase; SD, standard deviation.

there was no obvious change of grey value of craniocerebrum ultrasonic detection in the control group $(\mathrm{P}>0.05)$. The grey value of craniocerebrum ultrasonic detection in the observation group was significantly reduced at 7 and 14 days after birth (Table III).

Comparison of IL-6 levels of the children in the two groups showed that at 7 and 14 days after birth, the IL- 6 levels of both groups were significantly reduced, compared to the 1st day after birth, and the levels of the observation groups were significantly lower than that of the control group $(\mathrm{P}<0.05)$ (Table IV).
Comparison of NSE levels of the children in the two groups showed that at 7 and 14 days after birth, the NSE levels of both groups were significantly reduced, compared to the 1st day after birth, and the levels of the observation group were significantly lower than those of the control group $(\mathrm{P}<0.05)$ (Table V).

Comparison of S100 $\beta$ levels of children in the two groups showed that at 7 and 14 days after birth, the $\mathrm{S} 100 \beta$ levels of both groups were significantly reduced, compared to the 1st day after birth, and the levels of the observation 
Table VI. Comparison of S100 $\beta$ levels of child patients in the two groups $(\mathrm{ng} / \mathrm{ml}$, mean $\pm \mathrm{SD})$.

\begin{tabular}{lccrr}
\hline Group & $\mathrm{n}$ & 1 day after birth & 7 days after birth & 14 days after birth \\
\hline Observation group & 38 & $132.89 \pm 3.23$ & $115.52 \pm 3.15^{\mathrm{a}}$ & $94.83 \pm 3.04^{\mathrm{a}}$ \\
Control group & 38 & $132.36 \pm 3.28$ & $123.74 \pm 3.47^{\mathrm{a}}$ & $117.79 \pm 3.15^{\mathrm{a}}$ \\
t value & & 0.710 & 10.812 & 32.331 \\
P-value & & 0.480 & $<0.001$ & $<0.001$ \\
\hline
\end{tabular}

${ }^{\mathrm{a}} \mathrm{P}<0.05$, compared to the 1 st day after birth. $\mathrm{SD}$, standard deviation.

Table VII. Comparison of the DQ condition between child patients in the two groups (mean \pm SD).

\begin{tabular}{lcccccc}
\hline Group & $\mathrm{n}$ & Social adaptation & Gross motor & Fine motor & Language & Personal social contact \\
\hline Observation group & 38 & $86.73 \pm 5.05$ & $85.43 \pm 5.63$ & $77.23 \pm 3.56$ & $86.58 \pm 3.57$ & $86.58 \pm 3.57$ \\
Control group & 38 & $72.24 \pm 5.37$ & $71.86 \pm 5.78$ & $59.56 \pm 3.47$ & $70.56 \pm 3.48$ & $69.56 \pm 3.48$ \\
t value & & 12.117 & 10.367 & 21.911 & 19.808 & 21.045 \\
P-value & $<0.001$ & $<0.001$ & $<0.001$ & $<0.001$ & $<0.001$ \\
\hline
\end{tabular}

DQ, developmental quotient; SD, standard deviation.

Table VIII. Comparison of sequel incidence between child patients in the two groups [n (\%)].

\begin{tabular}{|c|c|c|c|c|c|c|}
\hline Group & $\mathrm{n}$ & $\begin{array}{l}\text { Cognitive } \\
\text { disturbance }\end{array}$ & $\begin{array}{l}\text { Cerebral } \\
\text { palsy }\end{array}$ & $\begin{array}{l}\text { Intelligence development } \\
\text { retardation }\end{array}$ & $\begin{array}{l}\text { Mental } \\
\text { retardation }\end{array}$ & Total \\
\hline Observation group & 38 & $2(5.26)$ & $1(2.63)$ & $1(2.63)$ & $1(2.63)$ & $5(13.16)$ \\
\hline Control group & 38 & $5(13.16)$ & $4(10.53)$ & $4(10.53)$ & $5(13.16)$ & $18(47.37)$ \\
\hline$\chi^{2}$ value & & & & & & 0.325 \\
\hline P-value & & & & & & 0.003 \\
\hline
\end{tabular}

group were significantly lower than that of the control group $(\mathrm{P}<0.05)$ (Table VI).

Comparison of the DQ condition at 12 months after treatment between the children in the two groups showed that the observation group scored significantly higher than the control group in the aspects of social adaptation, gross motor, fine motor, language, and personal social contact $(\mathrm{P}<0.05)$ (Table VII).

Comparison of the sequel incidence between the children in the two groups showed that the sequel incidence of the observation group was significantly lower than that of the control group $(\mathrm{P}<0.05)$ (Table VIII).

\section{Discussion}

White matter damage of premature infants is one of the most common causes of cerebral palsy, audiovisual obstacles, intelligence development retardation, epilepsy, learning difficulty and other brain damage syndromes, which seriously influence their quality of life (10). Prior studies have shown that there is a close relation between the occurrence of newborn brain damage and the fetal age (especially related to the low cerebrovascular developmental maturity of premature infants) (11). White matter damage of premature infants is caused by various factors, such as perinatal hypoxia and ischemia, developmental immaturity of brain structure, insufficient endocrine hormone as a result of getting out of matrix and placenta too early, perinatal infection, inflammatory reaction and deviation of neurodevelopment (12). At present, the pathogenesis of white matter damage of premature infants is not fully known. Usually, hypoxia and ischemia are taken as one of the main causes of the disease. The cerebrovascular developmental immaturity of premature infants can cause ischemic injury. In the process of damage, free radical damage, glutamate-induced excitotoxicity, microglia inflammation immunologic reaction and other damage mechanisms occur and finally lead to liquefaction and volume reduction of white matter (13).

Even though most treatments are still at exploration stage, the current treatments for the white matter damage of premature infants are the following: medicine intervention (brain active element, neurotrophic factor, erythropoietin, ganglioside and deferoxamine) and neural stem cell transplantation via ventricle (14). Results of the present study showed that 
the total effective rate of the observation group was higher than that of the control group $(\mathrm{P}<0.05)$. This might be due to the ganglioside capability to promote recovery after central nervous system damage. It might also affect the generation of cell transmembrane signal, interact with extracellular information and exert the effect of stabilizing membrane structure and function. Besides, ganglioside can prevent the influx of $\mathrm{Ca}^{2+}$, reduce the concentration of free radicals, reduce free radical damage, antagonize excessive activation of glutamate receptor, and reduce the damage to precursor cells of oligodendrocytes (15). Moreover, ganglioside can strengthen neurotrophic factors, and promote neuron regeneration.

Craniocerebrum ultrasonic detection has a high clinical diagnostic value and can be used to continuously and dynamically observe the condition of white matter of premature infants. This technique uses a sound wave with short pulse and high frequency and after reflection, refraction dispersion and absorption forms ultrasonic images on the oscilloscope screen. It has the advantages of easy operation and does not require the patient to move, and it is of high clinical diagnostic value (16). Results of this research showed that at 7 and 14 days after birth, the gray value of craniocerebrum ultrasonic detection of the control group showed no obvious change $(\mathrm{P}>0.05)$. At 7 and 14 days after birth, the gray value of craniocerebrum ultrasonic detection of the observation group was significantly reduced $(\mathrm{P}<0.05)$. This indicated that after ganglioside treatment, the echo of white matter around ventricle of the children got weaker and the damage degree of the children was well controlled and recovered.

IL-6 is a multi-functional cytokine related to inflammatory regulation and immune reaction, and it can take part in the inflammatory reaction of human body. It is produced by the astrocytes and microglias which are distributed in hippocampus, hypothalamus, cortex and other regions (17). Results of the present study showed that at 7 and 14 days after birth, the IL- 6 of the children in the two groups were significantly reduced, compared to the 1st day after birth, and the levels in the observation group were significantly lower than that of the control group $(\mathrm{P}<0.05)$. This might be due to the fact that in premature infants suffering from white matter damage, the brain tissue cells keep secreting IL-6 and local inflammatory reaction occurs. In this case, the intracranial pressure is increased and as the treatment time passes the concentration of IL-6 is reduced to levels below normal level, exerting central immune mediation, nerve repair and other physiological effects.

$\mathrm{S} 100 \beta$ protein is one of the acid calcium-binding proteins in glial cells. At normal condition, $\mathrm{S} 100 \beta$ can pass renal metabolism but not the blood-cerebrospinal fluid barrier. When brain damage occurs, blood-brain barrier can be destroyed and its permeability can increase. S100 $\beta$ can enter blood circulation by blood-brain barrier. Therefore, the level of $\mathrm{S} 100 \beta$ can be taken as the indicator to diagnose brain damage (18). NSE is a dimer-enzyme, often found in neuron cytoplasm, and when neuron damage occurs, NSE is released to extracellular fluid (19). Our results showed that at 7 and 14 days after birth, S100 $\beta$ and NSE levels in both groups were significantly reduced, compared to the 1st day after birth, and the levels of the observation group were significantly lower than that of the control group $(\mathrm{P}<0.05)$.
This may be due to the fact that ganglioside can activate the production of membrane lipids, stabilize the structure and function of cell membrane and directly exert a neuroprotective effect in brain, resulting in reduction of the brain damage (20).

After 12 months, the total effective rate of the observation group was higher than that of the control group. The observation group scored significantly higher DQ than the control group in the aspects of social adaptation, gross motor, fine motor, language and personal social contact. The sequel of patients in the observation group was significantly lower than that of the control group $(\mathrm{P}<0.05)$. This is because conventional treatment can hardly reverse the brain damage in premature infants. The efficacy is not obvious and tends to leave sequels. While the ganglioside can provide essential neurenergen for the development of the children, so that rich neural network may be developed in their brains, repair and promote the development of brain nerves again. Therefore, the normal intelligence development of the children is secured.

We conclude that the intervention treatment with ganglioside for premature infants suffering from white matter damage is beneficial and provides a protective effect. It also reduces sequel and produces promising results.

\section{Acknowledgements}

Not applicable.

\section{Funding}

No funding was received

\section{Availability of data and materials}

The datasets used and/or analyzed during the current study are available from the corresponding author on reasonable request.

\section{Authors' contributions}

XW collected and analyzed the general information of patients and wrote the manuscript. XT and JM interpreted the ultrasonic detection results. JZ assisted with white matter damage evaluation. All authors read and approved the final manuscript.

\section{Ethics approval and consent to participate}

The study was approved by the Ethics Committee of Tianjin Central Hospital of Obstetrics and Gynecology (Tianjin, China) and informed consents were signed by the guardians of the child patients.

\section{Patient consent for publication}

Not applicable.

\section{Competing interests}

The authors declare that they have no competing interests. 


\section{References}

1. Back SA: White matter injury in the preterm infant: Pathology and mechanisms. Acta Neuropathol 134: 331-349, 2017.

2. Wu CJ, Wang ZY, Yang YX and Luan Z: Long-term effect of oligodendrocyte precursor cell transplantation on a rat model of white matter injury in the preterm infant. Zhongguo Dang Dai Er Ke Za Zhi 19: 1003-1007, 2017 (In Chinese).

3. Smyser TA, Smyser CD, Rogers CE, Gillespie SK, Inder TE and Neil JJ: Cortical gray and adjacent white matter demonstrate synchronous maturation in very preterm infants. Cereb Cortex 26: 3370-3378, 2016.

4. Heese K: Functional repertoire of interleukin- 6 in the central nervous system - a review. Restor Neurol Neurosci 35: 693-701, 2017.

5. Erdem AF, Sahin YN, Dogan N, Umudum Z, Bayar F, Bulut C, Alici HA, Erkut B, Cesur M and Ceviz M: Effects of sevoflurane and propofol on $\mathrm{S} 100 \beta$ and neuron-specific enolase protein levels during cardiopulmonary bypass. Niger J Clin Pract 19: 278-283, 2016.

6. Kim DS, Son KY, Koo KM, Kim JY, Alfajaro MM, Park JG, Hosmillo M, Soliman M, Baek YB, Cho EH, et al: Porcine sapelovirus uses $\alpha 2,3$-linked sialic acid on GD1a ganglioside as a receptor. J Virol 90: 4067-4077, 2016.

7. Shin SH, Kim EK, Yoo H, Choi YH, Kim S, Lee BK, Jung YH, Kim HY, Kim HS and Choi JH: Surgical necrotizing enterocolitis versus spontaneous intestinal perforation in white matter injury on brain magnetic resonance imaging. Neonatology 110: 148-154, 2016.

8. Martinez-Biarge M, Groenendaal F, Kersbergen KJ, Benders MJ, Foti F, Cowan FM and de Vries LS: MRI based preterm white matter injury classification: The importance of sequential imaging in determining severity of injury. PLoS One 11: e0156245, 2016.

9. Zhang TW, Lin FT, Song YY, Wang LX and Cai YJ: Early intellectual developmental outcome of late preterm infants. Zhongguo Dang Dai Er Ke Za Zhi 19: 142-146, 2017 (In Chinese).

10. Su X, Tang W, Luan Z, Yang Y, Wang Z, Zhang Y, Wang Q, Suo L, Huang Z, Wang X, et al: Protective effect of miconazole on rat myelin sheaths following premature infant cerebral white matter injury. Exp Ther Med 15: 2443-2449, 2018.
11. Cai S, Liu Z, Peng G, Huang X, Li Y and Hu S: Therapeutic effects on cerebral white matter injury of premature infants treated with acupuncture for promoting the governor vessel and tranquilizing the mind. Zhongguo Zhen Jiu 38: 51-54, 2018 (In Chinese).

12. Wang LY, Tu YF, Lin YC and Huang CC: CXCL5 signaling is a shared pathway of neuroinflammation and blood-brain barrier injury contributing to white matter injury in the immature brain. J Neuroinflammation 13: 6, 2016.

13. Jadavji NM: The integrated stress response is not a target for diffuse white matter injury in premature infants. J Neurosci 37 : 11772-11773, 2017.

14. Junxiu LU, Bingyan Y, Jingguo C, Huanli XU and Haiyan C: Effect of cerebral hemodynamic changes on white matter damage in premature infant with patent ductus arteriosus. Zhejiang Da Xue Xue Bao Yi Xue Bani 45: 287-291, 2016 (In Chinese).

15. Schnaar RL: Gangliosides of the vertebrate nervous system. J Mol Biol 428: 3325-3336, 2016.

16. Tann CJ, Nakakeeto M, Hagmann C, Webb EL, Nyombi N, Namiiro F, Harvey-Jones K, Muhumuza A, Burgoine K, Elliott AM, et al: Early cranial ultrasound findings among infants with neonatal encephalopathy in Uganda: An observational study. Pediatr Res 80: 190-196, 2016.

17. Vasiljevic B: Neonatal hypoxic-ischemic brain injury. BMJ 340: 561-562, 2017.

18. Thompson WH, Thelin EP, Lilja A, Bellander BM and Fransson P: Functional resting-state fMRI connectivity correlates with serum levels of the S100B protein in the acute phase of traumatic brain injury. Neuroimage Clin 12: 1004-1012, 2016.

19. Yang L, Li D and Chen S: Hydrogen water reduces NSE, IL-6, and TNF- $\alpha$ levels in hypoxic-ischemic encephalopathy. Open Med (Wars) 11: 399-406, 2016.

20. Li L, Tian J, Long MK, Chen Y, Lu J, Zhou C and Wang T: Protection against experimental stroke by ganglioside GM1 Is associated with the inhibition of autophagy. PLoS One 11: e0144219, 2016.

This work is licensed under a Creative Commons Attribution-NonCommercial-NoDerivatives 4.0 International (CC BY-NC-ND 4.0) License. 